\title{
SIMULATION ANALYSIS OF SOLAR POWER GENERATION MICROGRID UNDER HUMAN-COMPUTER INTERACTION
}

\author{
Feng Tian ${ }^{1}$ \\ ${ }^{1}$ Shenyang Institute of Engineering, Shenyang, Liaoning, 110136, China. \\ Email: tianfengtianf2001@163.com
}

\begin{abstract}
This research aims to realize the effective control of solar power generation system by computer technology, and ensure the reliability of the link between microgrid and large grid. In this study, the man-machine interaction mode of the whole solar power system is realized by the virtual reality technology. The overall design of microgrid virtual reality technology for solar power generation system is introduced in this study. The environment around the microgrid of solar power generation system is simulated by 3DS Max virtual modelling tool. The radiation of the virtual solar power generation system is simulated by Matlab simulation and the special curve of solar cell I-V is also analysed by it. The results show that the 3D physical model of microgrid solar photovoltaic power generation is established by 3DSmax, and the model is exported to VRML by the Export function. Finally, the generated .wrl file in the text editor is opened, and the file is programmed and processed by the virtual reality language. The man-machine interaction between photovoltaic power generation system and human is realized by virtual technology, and the process of solar power generation is vividly presented by it. The simulation results of the solar power generation system show that the I-V situation of each node and the power status in the solar photovoltaic power generation process is observed by the I-V output characteristic curve. Therefore, the most efficient photovoltaic power generation system is designed by the curve, and the capacity of solar cells are selected and the appropriate battery capacity is chosen by it. Moreover, the reliability of power supply is further guaranteed. The research results have great significance for the realization of remote automatic control and management of micro power grid of solar power generation system in China.
\end{abstract}

Keywords: Human-Computer Interaction; Solar Power System; Microgrid; Virtual Reality Technology; Output Characteristic Curve.

\section{Introduction}

Since the 1980s, the power technology and internet network technology is developed rapidly in China. The conventional form of power generation, and centralized power generation and long-distance transmission of large internet network systems are formed by it [1]. Due to the rapid development of China's economy, the load of the end power grid is heavier and the stability and security of power grid operation are also affected. There are a large number of power outages during a natural disaster or emergency. The shortcomings of the existing power system are exposed by this phenomenon [2].

The power quality requirements of power system users of distributed generation technology are combined with the development trend of power system by some developed countries, and a set of special grid form microgrid is developed. The microgrid is connected with the large power grid, therefore, the electricity and heat is provided, and no environmental pollutant is produced, and the goal of environmental protection is achieved. At the same time, the large power grid the sustainable power supply of the large power grid is implemented [3].

A good solution for the power supply of natural disasters and emergencies is provided, and the power quality and safety of users are guaranteed. The power sources in the microgrid are various. Beside the traditional thermal power generation, the new energy is used, such as solar energy, wind energy, tidal energy fuel cells and so on.

These new energies are characterized by renewable energy, with inexhaustible reputation [4].

With the development of industrialization, the consumption rate of traditional energy, such as coal, oil and natural gas, has far exceeded the expected consumption rate. And most conventional energy sources are non-renewable. To ensure the normal progress of human daily life and industrial activities, it is urgent to find new energy sources to replace non-renewable energy sources. Among them, solar energy is one of the energy sources without pollution, and the technology of using solar energy to generate electricity is applied on a large scale [5]. Although solar power has many benefits, problems 
are still existed such as wide occupation area of the equipment, low energy conversion efficiency, and vulnerability to wind and temperature. Therefore, the normal operation of electrical equipment, convenience and the reduction of the cost are included in the design of solar power generation system, and some of its own problems need to be overcome. Thereby, it is necessary to design a set of solar power generation system reasonably.

In conclusion, to solve some problems in the microgrid system of solar power generation, solar photovoltaic power generation was simulated by virtual reality technology in this study.

Firstly, the overall design process of solar power generation microgrid system, and the amount of radiation on the array surface of a solar power generation microgrid system, and data calculation of battery I-V output characteristics were introduced.

Then through the simulation experiment, the realization of solar power generation microgrid simulation based on human-computer interaction was studied. Simultaneously, the radiation simulation results of solar photovoltaic power generation microgrid system and the simulation results of solar cell I-V output characteristics were analysed.

The research of this paper can provide a good idea for the diversification and intelligentization of power grid technology development.

\section{Literature Review}

With the development of the global economy, the industrial field is greatly developed, of course, the power technology is no exception. But the thermal power generation is adopted by the traditional power technology, which can cause some pollution to the air.

The decline of conventional energy is also a looming problem. Besides, large-scale power outages are caused by the traditional mode of power supply in the event of extreme weather and emergencies. To solve the above problems, relevant research work is carried out by scholars in various countries. Solar power generation is one of the new energy sources sought by scientists. Relevant literature reported that photovoltaic power generation has accounted for $87.9 \%$ of solar power generation [6].

Multi-objective particle swarm was used by Indragandhi et al. (2018) to optimize the usage of renewable resources. Thereby, the charging state of the battery was maintained, and some problems with fuel cells were solved. The minimum power exchange rate between (alternating current) AC/ (direct current) DC microgrids was defined. Solar and wind power were used as key resources in the system. Maximum utilization of resources was ensured by administrators and the power exchanges between microgrids were managed by maintaining charging status.
The results showed that the positive performance was achieved through multi-objective particle swarm optimization.

The electrical system proposed in this study was recommended as the best alternative to improve the utilization of electricity in remote areas [7].

A digital physical hybrid real-time simulation platform for power systems was proposed by Feng et al. (2018). The embedded physical system was combined with a digital simulator by this platform.

And the man-machine interface and data simulation systems were built by MFC, Matlab and other software. At the same time, the data exchange process of the active power system is simulated by embedded device. The close results produced by the simulation system were allowed, thus the advanced control strategies and algorithms were validated. With the development of computer technology, the internet of things technology was applied to the study of power grid by many scholars [8].

Internet of things technology was used by Prabha et al. (2018) to enhance the control of solar power generation load. The study pointed out that solar power was one of the fastest growing and most beneficial renewable energy sources in the world. Nowadays, the demand for electricity is increased in our daily life. In the long run, solar has the greatest potential and is expected to play an important role in the coming years. Two power supply modes were implemented. One was the normal mode of power supply, and the other was solar power generation. Computer or phone was used to control the internet of things, then the control of the operating load of the power system was achieved [9]. With the development of research, a growing number of high-tech technologies was introduced into solar power generation micro rid by scholars.

A coordinated control method of virtual power station was proposed by Liu et al. (2015) in research.

The photovoltaic power system and the controllable load were included in this method. Hence, the total power output of the virtual power plant was flexibly adjusted over a large range.

The hybrid integer programming problem was solved, and the power output of the photocell and the operating mode of the controllable load was coordinated. Effectiveness of frequency support of the island microgrid was provided by VPP, and it was verified by simulation results [10].

In conclusion, new sources of energy are found by scholars to replace the shortage of fuel, therefor, the problems faced by traditional electricity is solved. In this study, solar power generation is adopted according to previous researches.

To achieve the goal of human-computer interaction in the optimization of solar microgrid power generation system, it is necessary to apply the current new computer technology to solar power generation microgrid system. Regarding the characteristics of virtual reality technology, the 
virtual reality technology is used to study the simulation of solar power generation microgrid based on human-computer interaction in this study.

\section{Methodology}

\subsection{The overall design of virtual reality technology for solar power generation microgrid system}

To realize the man-machine interaction of solar power generation system, the virtual scene of microgrid solar photovoltaic power generation is constructed by virtual reality technology.

A virtual power generation platform is provided for users by the adoption of virtual technology.

And the goal of human-computer interaction is achieved by the means of virtual reality.

Changes in parameters such as voltage and current are obtained through virtual monitoring technology and simulation, and these changes are monitored.

The efficiency of solar photovoltaic power generation is controlled remotely by computer.

The main functions of the system are shown in Figure 1.

In the man-machine interaction stage, two functions of power generation virtual scene and power generation equipment monitoring platform are realized.

Meanwhile, the system parameter simulation and power generation equipment control are realized through the monitoring platform of power generation equipment.

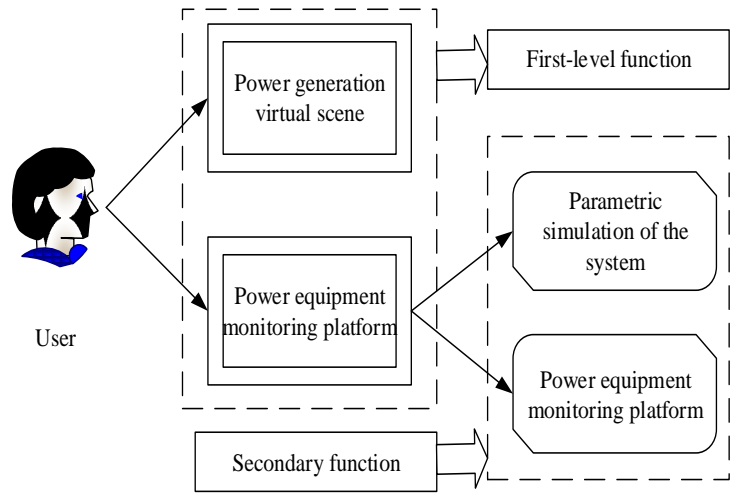

Figure 1: The main functions of solar photovoltaic power generation system in microgrid

Figure 2 is the virtual reality system operation diagram of solar power generation microgrid system designed in this paper.

Through the interaction of users and virtual scene, the control site in the virtual scene is accessed when the virtual scene of solar power generation microgrid is under the thumb. For example, realtime roaming of the virtual scene is operated on the keyboard. In the virtual scene, the workflow of power generation, real-time data generated, relevant parameters of power generation and running state of power generation equipment are realized.

The virtual object of power generation is controlled through the mouse, thereby, the process of solar power generation is under the thumb. During the monitoring of the field real-time data, the virtual display device is read directly through the display of the simulation model.

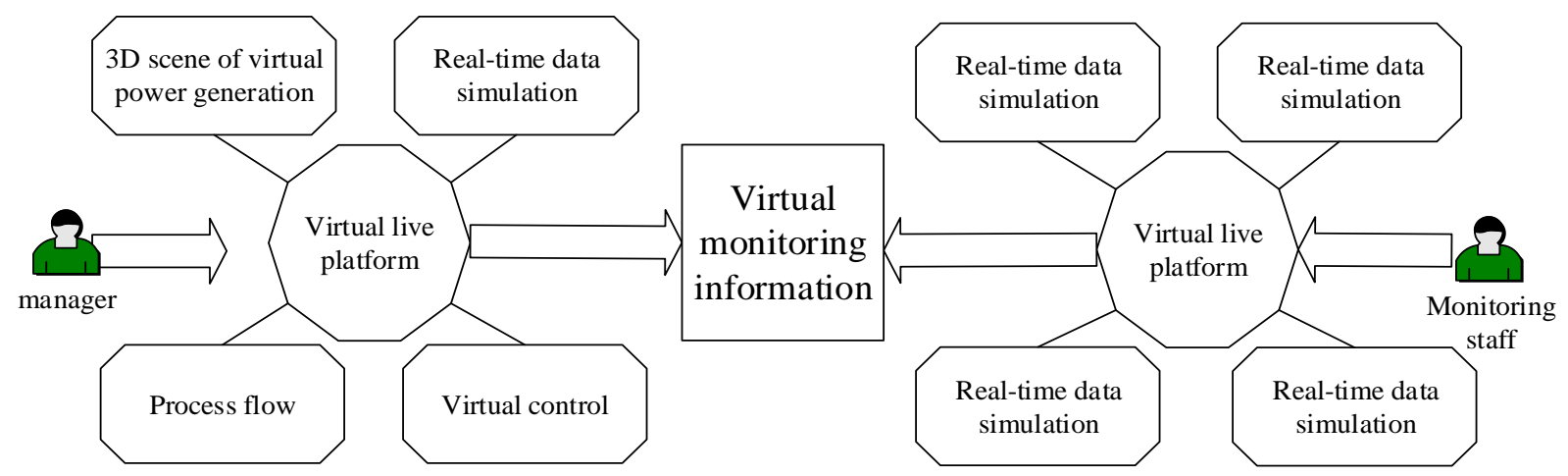

Figure 2: The virtual reality system operation diagram of solar power generation microgrid system

Solar power generation microgrid system based on human-computer interaction is a modern computer management technology, which integrates power generation technology with computer technology.

In this system, power operators are regarded as management users, and the virtual reality technology in computer technology is adopted to simulate the operation process of the whole solar photovoltaic microgrid system.
Computer control in the virtual environment is realized by computer virtual reality technology. Besides, it is necessary to train employees who use the system, adopt modern education methods, and build an open teaching system. Hence, the operation and control of the computer in a simple and fast way is realized, and human-computer interaction in a three-dimensional virtual environment is achieved.

According to the design goal of solar power generation microgrid simulation system, the overall construction is designed and analysed. 
Interactive convenience is the overall design goal of solar power generation microgrid simulation system based on human-computer interaction.

Figure 3 is the overall structure diagram of the microgrid simulation of solar power generation constructed according to the design objective in this paper.

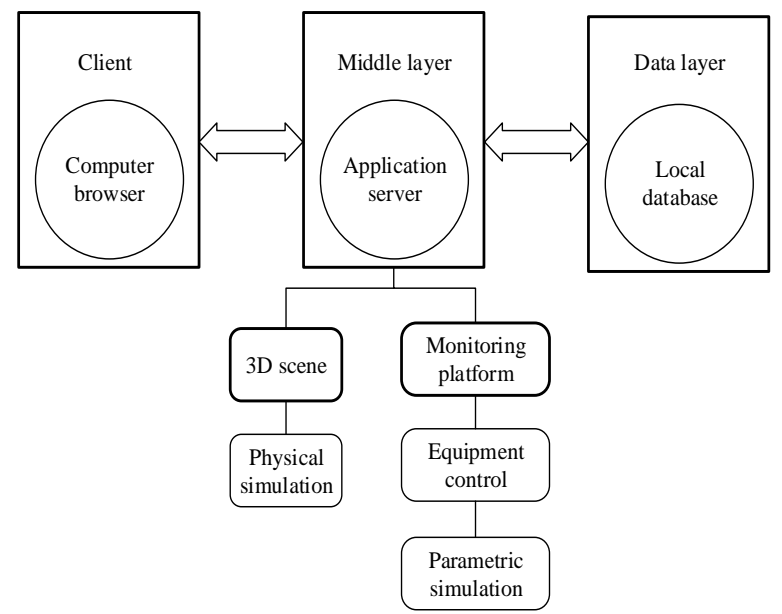

Figure 3: The overall structure diagram of the virtual reality system of solar photovoltaic power generation system

As shown in Figure 3, the customer layer, the middle layer, and the data layer is concluded in the overall structure diagram of the virtual reality system of solar photovoltaic power generation system.

In the client layer, it is the design for the virtual interface implemented by the computer.

A computer browser is installed to operate the process, simulation parameters and control of microgrid power generation. Thereby, the solar photovoltaic microgrid system is accessed.

The middle layer is operated according to the requirements from the users.

The physical model of solar photovoltaic power generation is judged by the middle layer and the virtual reality interface is also constructed by it.

Finally, the process of power generation is controlled according to the simulation parameters of the system.

The data layer is a temporary "locale" for the store of (voltage, current, power and solar array angle) simulation parameters. It is equivalent to database and computer information storage medium.

\subsection{Simulation of microgrid environment of solar power system}

To realize the simulation of solar photovoltaic power generation microgrid with human-computer interaction, a miniature solar power generation scenario is constructed by 3DS Max virtual modelling tool.
In the virtual scene of microgrid solar photovoltaic power generation system, the surrounding environment of microgrid solar photovoltaic power generation is observed from different angles and directions in the virtual scene. In this scenario, a house is simulated.

The total area of the house is about 100 square meters. To simulate the microgrid, the house is equipped with TV sets, electric lights, air conditioners and other electrical equipment.

Solar panels are laid on the south side of the house. Figure 4 shows the solar panels for the south floor of the house.

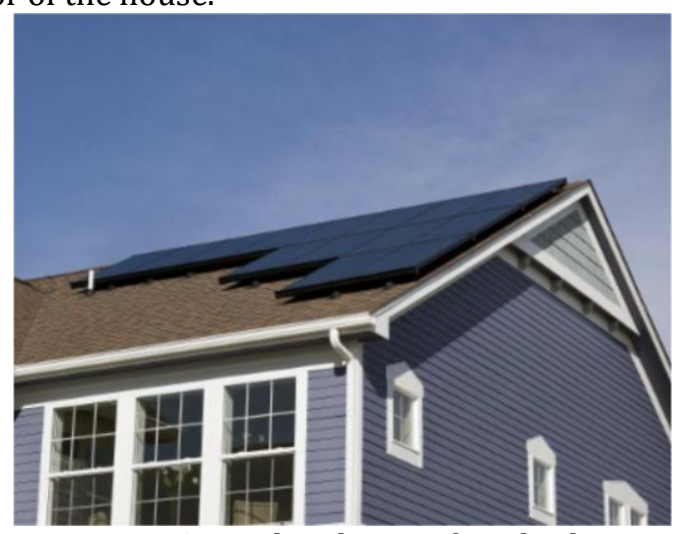

Figure 4: A simulated view of a solar house

As shown in Figure 4, there is no shielding in front of the solar panel. In this way, all the solar energy in the morning and afternoon is collected to maximize the charging time of the solar charging panel.

The work of the solar cell is affected by any shadow above the solar panel, and the normal operation of solar cells is damaged in severe cases.

Typically, there are no solar panels on the north side of the house.

The electricity generated by the solar system is connected to the large grid by smart meters and poles on the side of the house.

When sunlight hits a solar cell module in a power generation system, the solar energy is converted into electricity by the solar cell module.

It is effectively controlled by the controller under load conditions to charge the battery or directly power the load.

Besides, the inverter is also set up outside the house.

\subsection{Calculation and circuit simulation of solar power generation system}

According to the characteristics of solar cells, the amount of radiation from the battery array of solar photovoltaic power generation system is related to the apparent surface corresponding to the battery array.

Therefore, the installation of the solar lattice square array is selected in various directions, such as 
southward. It is also installed as a day tracking system (east-west tracking, horizontal axis tracking, full tracking, polar axis tracking).

The amount of solar energy emission is calculated according to different forms of motion. Hence, the mathematical model is established according to different forms of calculation.

Suppose the radiation received by the solar cell array every day is $F_{t}$, then:

$$
F_{t}=2 \int_{\omega_{\alpha}}^{0} F_{t}^{\prime} d \omega=2 \int_{\omega_{\alpha}}^{0} F_{0} F^{m} \cos \theta d \omega
$$

In this equation, solar constant is represented by $F_{0}, 1367 \mathrm{~W} / \mathrm{m}^{2}$ is taken here; and atmospheric transparency is represented by $F, m$ is the mass of atmosphere; $\theta$ is the Angle of direct sunlight, and $\omega$ is hour angle.

Suppose the scattered radiation received by the solar cell every day is $P_{t}$, then:

$$
P_{t}=2 \int_{\omega_{\alpha}}^{0} P_{t}^{\prime} d \omega=2 \int_{\omega_{\alpha}}^{0}\left[\frac{P_{p}^{\prime}\left(1+\cos \beta^{\prime}\right)}{2}\right] d \omega
$$

In this equation, $P_{t}$ represents the amount of scattered radiation that the horizontal plane can receive, and $\beta$ is the square inclination of the solar cell.

Suppose the reflection received form the ground is $Q_{T}$, then:

$$
Q_{t}=2 \int_{\omega_{\alpha}}^{0} Q_{t}^{\prime} d \omega=2 \int_{\omega_{\alpha}}^{0}\left[\frac{\lambda Q_{p}^{\prime}\left(1+\cos \gamma^{\prime}\right)}{2}\right] d \omega
$$

In this equation, ground reflectance is represented by $\lambda$, and $Q_{p}^{\prime}$ is the total irradiance on the horizontal surface.

The sum of the radiation amount on the inclined surface of the solar cell array is composed of the amount of radiation obtained daily, and the scattered radiation received daily, and the reflection accepted daily from the ground.

$$
W_{t}=F_{t}+P_{t}+Q_{t}
$$

According to the radiation calculation equation on the battery array of the solar energy photovoltaic power generation system in this section, the simulation circuit diagram is designed.

Figure 5 shows solar radiation intensity variation simulation module in one day.

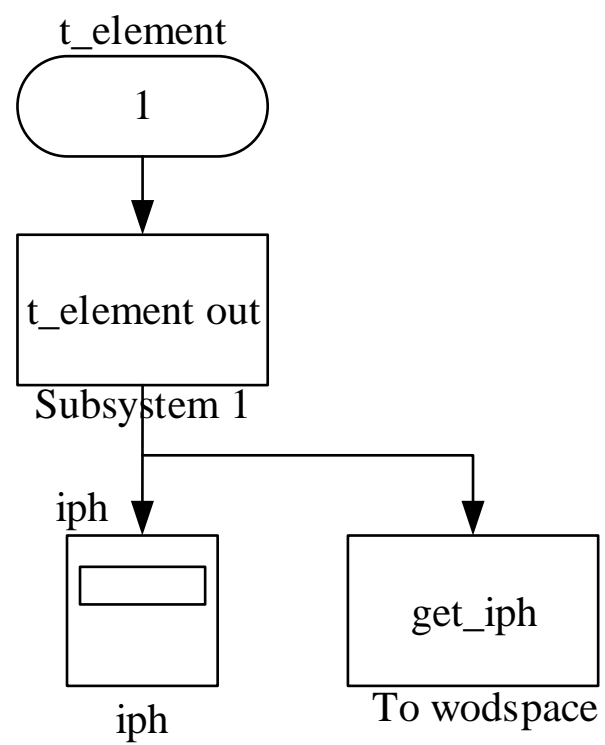

Figure 5: Simulation module design of solar radiation intensity variation in one day

\subsection{Mathematical calculation of the output characteristics of the I-V battery in a solar power generation system}

The electrical resistance is existed in the actual use of solar energy. Moreover, the effect of the resistance of the solar cell is taken into account. Without considering the effect of temperature on the solar I-V characteristic curve, the equivalent circuit of the actual solar cell is shown in Figure 6.

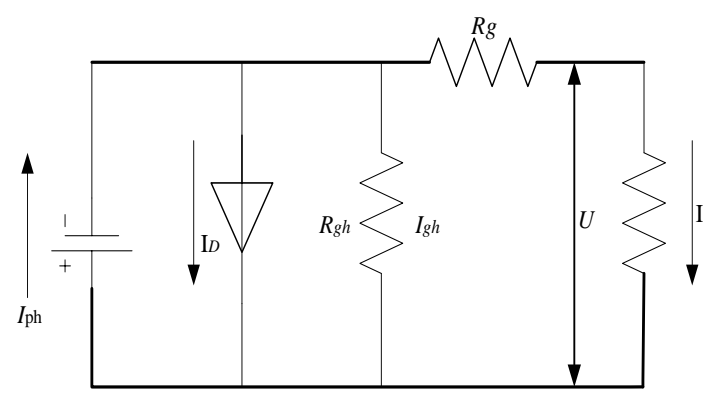

Figure 6: The equivalent circuit diagram of a solar cell

In the equivalent circuit, the voltage at both ends of the bypass resistor $R_{g h}$ is $U$.

$$
U_{j}=\left(U+I R_{\mathrm{g}}\right)
$$

Then the current at both ends of the bypass $R_{g h}$ resistance is:

$$
I_{g h}=\left(U+I R_{g}\right) / R_{g h}
$$

The passing load current is expressed as:

$$
I=I_{p h}-I_{D}-I_{g h}=I_{p h}-I_{0}\left(\mathrm{e}^{\frac{q\left(U+I R_{s}\right)}{A K T}}-1\right)-\frac{U+I R_{s}}{R_{s h}}
$$




\section{Results}

\subsection{Realization of microgrid simulation of solar power generation based on human- computer interaction}

This study aims to realize the man-machine interaction of solar power generation micro-grid simulation, Firstly, the simulation environment of virtual reality technology is analysed, because the study in this paper is to achieve the simulation of the whole process of solar photovoltaic power generation micro-grid. Therefore, the simulation of each process is analysed comprehensively.

Firstly, the background is added. To distinguish the sky and the ground under the solar irradiation, the panorama of the solar house and the sky are simulated with a realistic effect.

Then the viewpoint is added. The view node of browser is adopted to write a Viewpoint \{...\}in VRML language. In this way, different perspectives are selected to observe the virtual scene, and the power generation process in all directions of solar power generation microgrid is monitored. Hence, the realistic effect of man-machine interaction is achieved. Multi-dimensional perspective observation can't be achieved, when the traditional .wrl files format are used in viewing in the browser. And it is not very efficient. After the adoption of the browser view node, the high-efficiency multi-directional observation of the solar power generation simulation scene is realized.

Besides the addition of the background, the television (TV) set is simulated in virtual reality. The operation of the television is equivalent to a load in the microgrid of a solar power system. According to the rendered interface, when the sun appears in the morning, the microgrid solar power system starts to generate electricity. Moreover, When the electricity is turned on, the play mode of TV is opened. The virtual reality technology is used to animate the TV. Through the simulation of TV, the simulation of solar power generation micro grid is vividly presented to the staff.

The light and shade and color distribution on the surface of other objects is calculated by virtual reality technology. The object is contrasted with the surrounding environment to show the lighting effects. The sun's position in space and the emission direction of light is specified. And the color and brightness of the sun's rays are set and 3D model of the sun is built. Then set it as a parallel light source through the Directional Light node. Therefore, light for the entire virtual environment is provided. The light is emitted and spread out, when sunlight hits the panels.

The input-output state is defined by the routing settings in VRML. Hence, the sun in the virtual system is moved along a specific trajectory, and the hierarchy of the scene diagram is changed.
The whole process of the reception of the sun through the solar arrays is vividly represented. Moreover, the variation of solar radiation intensity is also displayed.

In conclusion, 3DSmax is used to build the $3 \mathrm{~d}$ physical model of microgrid solar photovoltaic power generation, the model is derived to the VRML by Export function, and finally open the generated .wrl file in the text editor. The file is programmed and processed through virtual reality language. Thereby, the man-machine interaction between photovoltaic power generation system and human is realized by virtual technology. The process of solar power generation is also vividly presented.

\subsection{Radiation simulation results of solar power generation microgrid system}

The solar radiation intensity and solar radiation accumulation of solar photovoltaic microgrid system are simulated in this study, the simulation results are shown in Figure 7. According to the results in Figure 7 , both solar radiation intensity and solar radiation accumulation are increased after 6 am. The maximum value of solar radiation is achieved around 14 o 'clock in the day. However, the solar radiation accumulation increased around 6 am. As shown in the simulation diagram, the increase is risen at a uniform rate until about $6 \mathrm{pm}$.

The solar energy radiation accumulates at a peak and does not change after sunset.

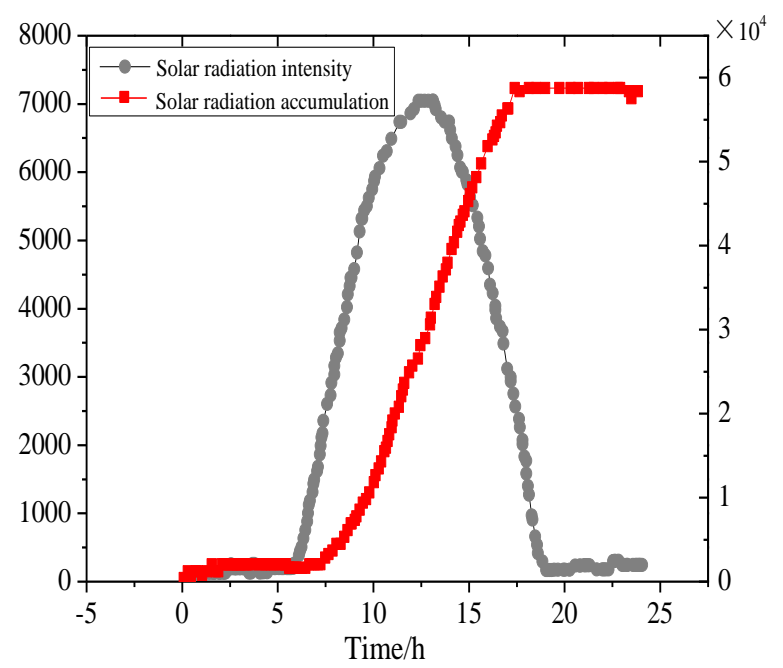

Figure 7: The curve of solar radiation intensity changes with time and the cumulative graph of solar radiation in a day

\subsection{Simulation results of I-V output characteristic curve of solar cell}

According to the mathematical calculation of I-V output characteristic of solar photovoltaic power generation system, the I-V characteristic curve is simulated. 
Figure 8 shows the comparison between the I-V characteristic curve and the ideal model, and Figure 9 shows the comparison between the P-V characteristic curve and the ideal model. As shown in the figure, in the I-V curve, only one of the measured values is almost similar to the results of the model, and the effect of the other measured values is far different from that of the ideal model with uniform values. However, in the P-V characteristic curve, all the measured results are quite different from the ideal model. Therefore, to provide the power of solar photovoltaic power generation, it is necessary to change various control strategies.

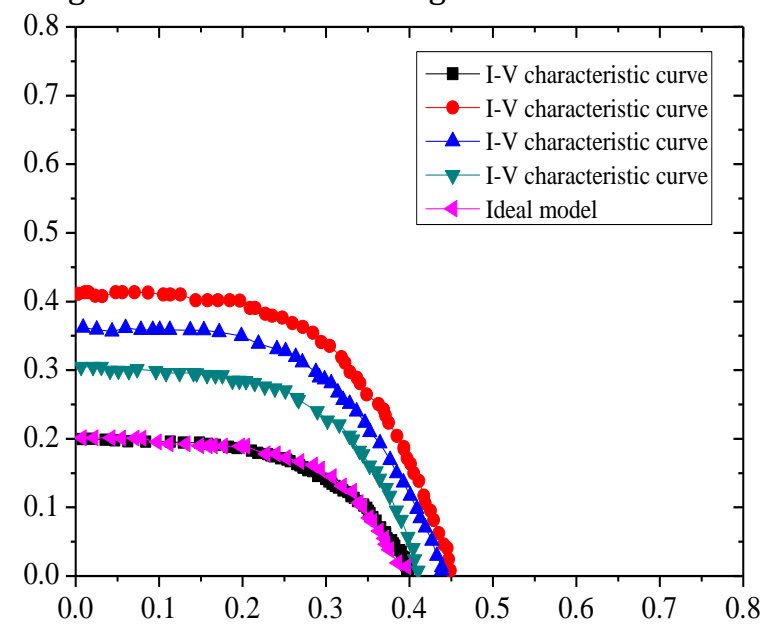

Figure 8: Comparison of I-V characteristic curve and ideal model

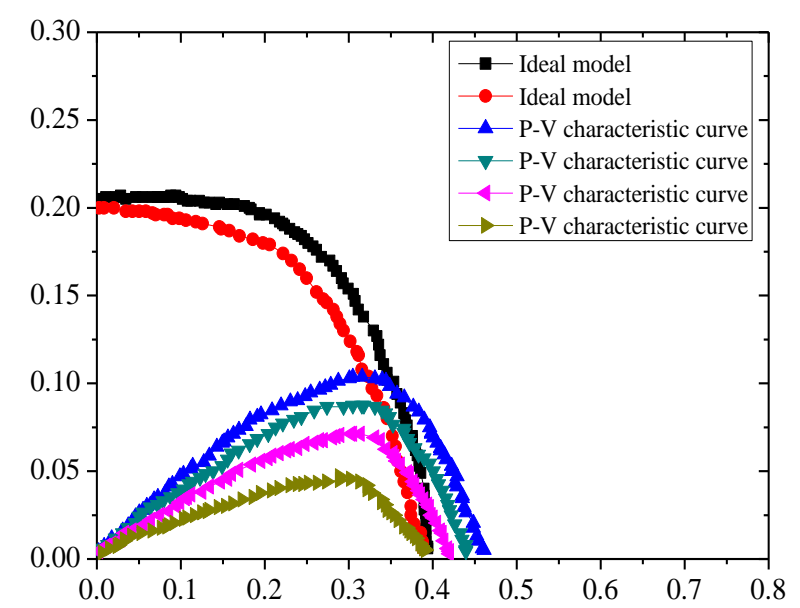

Figure 9: Comparison of P-V characteristic curve and ideal model

\section{Discussion}

This study aims to realize the simulation of solar power generation microgrid based on humancomputer interaction. The purpose of humancomputer interaction in power grid system is to realize the convenient management of power grid system and meet the demand of power grid durability, responsiveness and naturalness. This study shows that the interaction between the microgrid of solar power generation system and human is well realized by virtual reality technology. In the research of Sangi and Müller D (2018) [11], a compact wearable and low-power human-computer interaction device for gesture recognition is realized by the human-computer interaction technology. According to the operation of the DC microgrid and the capacity of the converter station, the DC microgrid with full power cage asynchronous wind generator, energy storage battery and AC-DC load is divided into five modes, namely grid-connected operation, current-limiting operation, short-time failure operation, island unloading operation and island power off operation. The power coordination control strategy is automatically coordinated the work of each substation according to the change of DC voltage, the charging state of the battery and the current of the grid-connected converter. The power balance of the power grid system and various of DC microgrid systems is an important guarantee for the safe and stable operation of the power grid. The research of Velmurugan et al. (2018) [12] is a practical operation of real power grid operation.

This study is in the stage of simulating power grid system, but the same results are obtained. When the power grid system is further simulated, the I-V output characteristic curve of the solar power generation system is obtained to observe the power status and the I-V situation of each node in the solar photovoltaic power generation process.

Therefore, the most efficient photovoltaic power generation system is designed, and a selection strategy for the capacity of solar cells is achieved, and the right battery capacity is chosen. The research of $\mathrm{Lu}$ et al. (2018) [13] shows that the power generation process of solar energy is well reflected by the I-V output characteristic curve, which has a good guiding significance for the selection of solar cells. Therefore, the research results of this study are consistent with the previous research results in theory.

The man-machine interaction of solar power generation micro-grid is realized, and the monitoring of solar power generation process and the selection of solar cell capacity are guided.

\section{Conclusions}

To realize the remote control and management of solar power generation system, the virtual reality technology is used. Hence, the simulation of solar power generation microgrid with human-computer interaction is realized. Firstly, the virtual reality technology used in solar power generation micro grid system is designed in this study, then the surrounding environment is simulated. The results show that the man-machine interaction between photovoltaic power generation system and human is realized by the 3DS Max virtual. Moreover, the process of solar power generation is vividly presented. The virtual solar power generation 
system and the I-V special curve of the solar cell is simulated and analyzed by the Matlab simulation, then the I-V output characteristic curve is obtained. Hence, the I-V situation and the power status of each node in the process of solar photovoltaic power generation are observed.

Therefore, the most efficient photovoltaic power generation system is designed, and a selection strategy for the capacity of solar cells is achieved, and the right battery capacity is chosen.

The results in this study made a good contribution to the realization of automatic monitoring and management of the microgrid of solar power generation system. the safety and accuracy of solar power generation is ensured. However, the research in this study still has limitations.

The realization of the connection between human-computer interaction and network is the focus of the next research.

\section{References}

[1] Liu Y., Gong M., Liang L, et al. (2018) Research and design of low-power grid-connected PV power generation system based on automatic solar tracking. Systems Science \& Control Engineering, 6(3), 278-288.

[2] Qingyu F., Wanneng Y., Junxian Z, et al. (2015) Research and development of solar power supply control system based on PLC. Automation \& Instrumentation, (9), 34.

[3] Park E., Cho Y., Han J, et al. (2017) Comprehensive approaches to user acceptance of Internet of Things in a smart home environment. IEEE Internet of Things Journal, 4(6), 2342-2350.

[4] Hussain A., Barakat M. M., Aliyu S, et al. (2019) Impact of usability assessing and testing on educational portal. Journal of Computational and Theoretical Nanoscience, 16(5), 2131-2134.
[5] Rafique S. F., Shen P., Wang Z, et al. (2018) Global power grid interconnection for sustainable growth: concept, project and research direction. IET Generation, Transmission \& Distribution, 12(13), 31143123.

[6] Zhang X., Yu T., Xu Z, et al. (2018) A cyberphysical-social system with parallel learning for distributed energy management of a microgrid. Energy, 165, 205-221.

[7] Indragandhi V., Logesh R., Subramaniyaswamy $\mathrm{V}$, et al. (2018) Multi-objective optimization and energy management in renewable based AC/DC microgrid. Computers \& Electrical Engineering, 70, 179-198.

[8] Feng X., Yan T., Zhang A, et al. (2018) A Matlabbased digital-physical hybrid real-time simulation platform for power system, IEEE, 12(3): 4253-4258.

[9] Prabha G., Mohana K. (2018) Design of feasible energy generation using solar panel and control using an IoT. International Journal of Engineering \& Technology, 7(24), 191-196.

[10] Liu Y., Xin H., Wang Z, et al. (2015) Control of virtual power plant in microgrids: a coordinated approach based on photovoltaic systems and controllable loads. IET Generation, Transmission \& Distribution, 9(10), 921-928.

[11] Sangi R., Müller D. (2018) A novel hybrid agentbased model predictive control for advanced building energy systems. Energy conversion and management, 178, 415-427.

[12] Velmurugan U., Francis D. J., Pradeep M. (2018) Realization of visual verification procedure by using QR code system. International Journal of Pure and Applied Mathematics, 119(15), 805811.

[13] Lu S., Li Y., Xia H. (2018) Study on the configuration and operation optimization of CCHP coupling multiple energy system. Energy conversion and management, 177, 773-791. 